\title{
Analytical study of care quality and moral distress in clinical situations and patient care
}

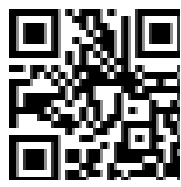

Original article

Masoumeh Hasanloa,*, Arezo Azarm ${ }^{\mathrm{b}}$ Parvaneh Asadic, Kourosh Amini ${ }^{\mathrm{d}}$, Hossein Ebrahimie, Mohammad Asghari Jafarabadif

${ }^{a}$ Department of Surgical Technology, Zanjan University of Medical Sciences, Zanjan, Iran

${ }^{b}$ Education and Treatment Center of Beheshti, Zanjan University of Medical Sciences, Zanjan, Iran

cKermanshah University of Medical Sciences, Kermanshah, Iran

'Zanjan Social Determinations of Health Research Center, Zanjan University of Medical Sciences, Zanjan, Iran

${ }^{e}$ Department of Psychiatric Nursing, Faculty of Nursing and Midwifery, Tabriz University of Medical Sciences, Tabriz, Iran

${ }^{t} H$ Health Services Management Research Center, Faculty of Public Health, Tabriz University of Medical Sciences, Tabriz, Iran

Received: 4 August 2018; Accepted: 5 November 2018; Published: 20 September 2019

Abstract: Objective: Nursing profession conventionally meets a high standard of ethical behavior and action. One of the ethical challenges in nursing profession is moral distress. Nurses frequently expose to this phenomenon which leads to different consequences such as being bored by delivering patient care that decline care quality and make it challenging to achieve health purposes. This study was conducted to investigate the association between the aspects of moral distress and care quality.

Methods: In this descriptive-analytical study, 545 nurses of intensive and cardiac care units and dialysis and psychiatric wards were recruited by census sampling. Three questionnaires, Sociodemographics, Moral Distress Scale, and Quality Patient Care Scale, were distributed among the participants and collected within 9 months. Data analysis was conducted by descriptive statistics, analysis of variance, and the least significant difference in SPSS 13.

Results: Investigating moral distress domains (ignoring patient, decision-making power, and professional competence) and care quality domains (psychosocial, physical, and communicational) demonstrated that in being exposed to moral distress, ignoring patient had no effect on psychosocial domain $(P=0.056)$, but decision-making and professional competence of moral distress had positive effect on psychosocial, physical (bodily), and communication domains of care quality.

Conclusions: Because moral distress domains are effective on patient care quality, it is recommended to enhance the knowledge of nurses, especially beginners, about moral distress, increase their strength alongside standardizing nursing services in decisionmaking domains, improve the professional competence, and pay attention to patients.

Keywords: quality of healthcare $\bullet$ moral distress $\bullet$ clinical situations $\bullet$ nurse $\bullet$ patients

(c) Shanxi Medical Periodical Press.

\section{Introduction}

Nursing profession meets a high standard of ethical behavior and action. ${ }^{1}$ The stipulated purposes in this profession are obviously ethical and seek to protect patients against injuries and complications and maintain the healing environment because most patients are

How to cite this article: Hasanlo M, Azarm A, Asadi P, Amini K, Ebrahimi H, Jafarabadi MA. Analytical study of care quality and moral distress in clinical situations and patient care. Front Nurs. 2019; 4: 327-334.

* Corresponding author.

E-mail: Hasanlo_md@yahoo.com (M. Hasanlo).

כ Open Access. ๑ 2019 Masoumeh Hasanlo et al., published by Sciendo. (c) BY-NC-ND This work is licensed under the Creative Commons Attribution NonCommercial-NoDerivatives 4.0 License. 
vulnerable and need complete and timely care. ${ }^{2}$ Moral distress, an important problem experienced by multiple healthcare disciplines, is defined variously in different studies. While there are many sources of stress in healthcare work, moral distress is specifically associated with the ethical dimensions of practice and concerns related to difficulties navigating practice while upholding professional values, responsibilities, and duties. ${ }^{3}$

Moral distress, regardless of being understood differently in different studies, has been shown to have negative consequences, contributing to emotional distress (e.g., anger and frustration), withdrawal of self from patients, unsafe or poor quality of patient care, decreasing job satisfaction, and even attrition in nursing. ${ }^{4}$ Today, nurses experience moral distress more frequently than in the past due to increased complication of healthcare system, clinical mistakes, and nursing understaffing. ${ }^{5}$

Caring capacities of the nurses decrease, they may fail to satisfy the patients' basic and physical needs, or evade being from the patient's bedside or nursing profession. ${ }^{6,7}$ Complying with patient's rights and needs along with the Nursing Code of Ethics are the pivotal component of care ${ }^{5}$ and the ultimate goal of nurse is to deliver high-quality care. ${ }^{8}$ In other words, receiving quality care is the right of all patients and the responsibility of all caring nurses. ${ }^{9}$

Burhans considers purposefulness to be the most important characteristic of quality of care that is realized by delivering the most optimal care. ${ }^{10}$ As nurses form the main core of the quality improvement process, their performance is very effective in advancing organizational goals.

Critical care unit nurses are facing environmental stress, heavy work load, understaffing, and difficult conditions that influence the process of nursing care and can undermine the quality of the delivered care. ${ }^{11}$ The factors such as clinical situation, patient care, and nurse contribute to development of moral distress in nursing. Patient care includes the decisions that are taken for the patient and he/she does not benefit from it. ${ }^{12}$ Meanwhile, moral distress is one of the effective factors on nursing care such that studies have indicated that as moral distress occurs, the nurses' caring capacities decline..$^{6,7}$

Given the wide variety of stressors in the nursing profession, ${ }^{13}$ their negative impacts on the quality of nurses' work including care for patients, ${ }^{14}$ psychological stresses related to moral distress among Iranian nurses, ${ }^{15}$ and to achieve a perfect and optimal level of quality, it is necessary to seek out novel solutions for promoting the quality of patient care. According to the available evidence, the domains of moral distress have not yet been investigated with reference to nursing care quality in Iran. Therefore, this study was conducted to investigate the association between moral distress domains and nursing care quality in psychiatric ward because nurses should report the patients' behaviors and performance for the physicians' appropriate decision-making and diagnosis and selecting the required nursing care in addition to pharmacotherapy as committing mistake in this process can cause distress and the inadequacy of patient care.

In the critical care units, the nurses should have up-todate knowledge and facilities to have appropriate performance, and it is efficient to monitor the patients, particularly younger ones, through the process of their recovery and saving, which helps to decrease the costs, advance healthcare system, and promote health index. This study was conducted in the teaching hospitals of Tabriz, Iran.

\section{Materials and Methods}

\subsection{Study design}

This descriptive-analytical study was conducted in intensive care units (ICUs), cardiac care units (CCUs), and dialysis and psychiatric wards.

\subsection{Setting and sample}

Because we found no similar study as far as we searched, with regard to the limited number of nurses, subjects included 602 nurses from Tabriz teaching hospitals who were selected by census sampling and conducted within 9 months from ICUs and CCUs, dialysis and psychiatric wards.

Because 23 questionnaires were not returned, 12 questionnaires were not completed because of lack of time and being busy, lack of familiarity with the statistical principles, and therefore lack of interest in research, the inability to use the results of research in nursing, managers' reluctance to hear employees' concerns, lack of useful and continuous training and promotion programs for employees, and personal reasons, and 22 people did not fulfill the inclusion criteria, 545 nurses were recruited via paper-and-pencil self-administered assessment in the study. The inclusion criteria were having bachelor's degree and over, having worked in the studied ward for at least 6 months, and being willing to participate in the study. After the Ethics Committee approved the study protocol, the Research Deputy provided the approval, the researcher prepared the questionnaires and determined the target centers, and then referred to the centers to distribute the questionnaires among the samples at morning, afternoon, and night shiftworks after making necessary coordinations with the head, nursing directors, and supervisors. Then, the completed questionnaires were collected within 3-7 days. 


\subsection{Ethical consideration}

This study was part of a research project, which has been approved by Tabriz University of Medical Sciences (approval no. 12331/4/5). To observe ethical considerations, the researcher introduced herself and emphasized that the data would be kept private, there would be no need for mentioning name in the questionnaire, the participation in the study would be voluntary, and the necessary explanations about the history and definitions of moral distress and the research purpose were delivered to the participants. In addition, the participants were ensured that they would be informed about the results of the study so that they would be able to offer their recommendations to improve care quality considering available scientific gap for conducting further studies and expending time and cost to draw the participants precision and honesty in replying to the items as much as possible. The respondents were instructed to leave the items on which they had no experience unanswered.

\subsection{Measures and data analysis}

Data collection was conducted by three questionnaires: Sociodemographics Questionnaire, Corely's Moral Distress Scale (MDS), and Quality Patient Care Scale (QUALPACS). In the present study, the validity of the MDS was investigated using the corrective comments of 12 professors of medicine, nursing and midwifery, community health, ethics, and psychology from the Tabriz University of Medical Sciences, and its reliability coefficient was derived 0.94 by internal consistency and the Cronbach's alpha coefficient. Data analysis was conducted by using SPSS 13 using descriptive statistics of frequency (\%) and mean (standard deviation [SD]) for the severity of distress and the level of care quality, and analytical statistics to study the relationship between three domains of moral distress and three domains of care quality.

To investigate the patient care quality, QUALPACS was used that has been frequently used in the United States since 1975 and occasionally used in the UK and Nigeria. This scale was translated into Persian 2010, Iran and its validity and reliability were confirmed. ${ }^{16}$ The QUALPACS consists of 68 items and assesses nursing care quality in three domains: psychosocial (32 items), physical (23 items), and relational (13 items).

In care quality section, the respondent replied to the items on psychosocial domain that were rated by 4-point Likert scale (from never to always) with 1 representing never, 2 representing rarely, 3 representing often, and 4 representing always. Because this domain consists of 32 items, its minimum and maximum possible scores are 32 and 128, respectively. For physical domain consisting of 23 items, the scores range between 23 and 92. For communication domain consisting of 13 items, the scores range between 13 and 52 .

MDS is extensively used to measure moral distress in nursing and was first used in $1995 .{ }^{17}$ Another study investigated the MDS validity in Iran using content validity, and its reliability was derived 0.93 by test-retest and Cronbach's alpha coefficient. ${ }^{18}$ This questionnaire consisted of 30 questions in three categories of ignoring patient (16 questions), decision-making power (8 questions), and professional performance competence (6 questions) which were rated by using 7-point Likert scale from very low to very high, number 1 represented the lowest moral distress, and number 7 represented the highest moral distress; as a result, the scores of the intensity of moral distress ranged between 30 and 210 .

The methods used in Likert questionnaires are nonparametric because the main nature of such questionnaires is qualitative and use of parametric methods can lead to wrong conclusions and, as you know, in the nonparametric methods, the real data are not used, and the power of data presentation reduces. Therefore, in analyzing the relationship between the dimensions of moral distress and care quality, a combination of parametric and nonparametric methods is used to maintain the data presentation power. It should be noted that the scores obtained for each questionnaire do not show the exact meaning of a continuous number and also should be a nonparametric viewpoint on it.

First, the dimensions of moral distress are classified into three classes according to the scores derived from Likert scales, which is due to variable formatting in application of relevant tests. It should be noted that the classification of the dimensions of moral distress cannot be understood as data presentation power reduces, because in any case, due to Likert scales nature, for analysis, nonparametric methods should be used, in which the original data are not available.

In this article, the dimensions of moral distress three classes were considered independent and the association of care quality domains with this variable was investigated. Because the questionnaire's items were rated by Likert scale, first, the variable was converted such that continuous data were used instead of discrete data. Therefore, if a person had severe or weak moral tension, care quality would not be the same.

Minimum and maximum possible scores on ignoring patient (n: 16) are 16 and 112, respectively. Then, this range of numbers was divided into three categories with equal length: The first category (16-48) represents low levels of ignoring patients, the second category (48-80) represents moderate levels of ignoring patient, and the third category (81-112) represents high levels of ignoring patient. Similarly, in decision-making domain 


\begin{tabular}{lccc}
\hline Items $(n)$ & $\begin{array}{c}\text { Low level } \\
(16-47)\end{array}$ & $\begin{array}{c}\text { Moderate level } \\
(48-80)\end{array}$ & $\begin{array}{c}\text { High level } \\
(81-112)\end{array}$ \\
\hline \hline $\begin{array}{l}\text { Ignoring patients } \\
\text { domain (16) }\end{array}$ & $\mathrm{A}$ & $\mathrm{B}$ & $\mathrm{C}$ \\
$\begin{array}{l}\text { Decision-making } \\
\text { domain (8) }\end{array}$ & $\mathrm{D}$ & $\mathrm{E}$ & $\mathrm{F}$ \\
$\begin{array}{l}\text { Professional } \\
\text { competence domain (6) }\end{array}$ & $\mathrm{G}$ & $\mathrm{H}$ & $\mathrm{K}$ \\
\hline Items & & Number of items & Item score \\
\hline \hline Psychosocial domain & & 32 & $32-128$ \\
Physical domain & & 23 & $23-92$ \\
Communication domain & & 13 & $13-52$ \\
\hline
\end{tabular}

Table 1. Moral Distress Scale 7-point Likert 30 questions

consisting of eight items, minimum and maximum possible scores for the decision-making are 8 and 56 , we had three categories, respectively; 8-23 to represent low power of decision-making, 24-39 to represent moderate power of decision-making, and 40-56 to represent high power of decision-making. In professional competence domain (six items) from a minimum possible score of 6 to a maximum possible score of 42 , the scores were divided into three categories, 6-17 representing low levels of professional competence, 18-30 representing moderate levels of professional competence, and $31-42$ representing high levels of professional competence (Table 1).

Finally, to further investigate and understand the relationship between moral distress domains and care quality domains, analysis of variance was used by means of new variables.

\section{Results}

In the studied wards of the 10 education treatment hospitals of Tabriz, $86.6 \%$ of the participants were women and $56 \%$ were married, and most of them had bachelor's degree and were working in Imam Reza and Madani healthcare centers (Table 2).

The mean score on moral distress in the ICUs/ CCUs and psychiatric wards was $141.89 \pm 29.6$ and within a moderate range with severe moral distress in $225(41.3 \%)$ participants and moderate moral distress in $288(52.8 \%)$ ones. The most important causes of moral distress among the nurses were understaffing (303 participants), delivering inadequate patient care (290 participants), lack of professional competence and ability to perform a duty (281 participants), ignoring caregivers' bothering patients (276 participants), and ignoring and lack of reporting the errors committed by nurses ( 275 participants) that were reported by over $50 \%$ of the samples considering the total number of them.

\begin{tabular}{lc}
\hline Sociodemographic characteristics of studied nurses & $n(\%)$ \\
\hline \hline Hospital & \\
Shohada & $28(5.1)$ \\
Alzahra & $15(2.8)$ \\
ShahidMadani & $150(27.5)$ \\
Children Cure \& Health Hospital & $47(8.6)$ \\
Alavi & $9(1.6)$ \\
Imam Reza & $160(29)$ \\
Taleghani & $13(2.9)$ \\
Nikoukari & $8(1.4)$ \\
Razi & $90(16.5)$ \\
Sina & $25(4.6)$ \\
Ward & \\
Special & $455(83.5)$ \\
Psychiatric & $90(16.5)$ \\
Gender & \\
Female & $472(86.6)$ \\
Male & $73(13.4)$ \\
Marital status & \\
Single & $239(43.9)$ \\
Married & $306(56.1)$ \\
Education & \\
Bachelor & $511(93.8)$ \\
Master \& Higher & $34(6.2)$ \\
Occupation & \\
Employed & $157(28.8)$ \\
Contract and Project & $388(71.2)$ \\
\hline & \\
\hline
\end{tabular}

Table 2. The sociodemographic characteristics of studied nurses

The mean score on care quality was $195.97 \pm 28.05$. In all, $202(37.1 \%)$ participants reported care quality to be relatively desirable and $333(61.2 \%)$ reported it to be desirable. Care quality at patient request for meeting a clergyman (244 participants), introducing new patient to the room patients (242 participants), familiarizing the patients with similar problems for sympathy (240 participants), using fragrant substances to freshen the environment (209 participants), and nurses' instructing and paying attention to religious ordinances in the case of physical problems in the patient (205 participants) were reported to be low comprising over onethird of the samples.

Although moral distress was severe in 225 participants, that is, over one-third of the study samples, the results on care quality were desirable. This finding was investigated using Pearson correlation analysis.

Pearson correlation analysis showed that moral distress and nursing care quality were not significantly associated $(P=0.058)$. To better predict the relationships among low, moderate, and high levels of moral 


\begin{tabular}{|c|c|c|c|c|c|c|c|c|c|}
\hline \multirow[t]{2}{*}{ Items } & \multicolumn{3}{|c|}{ Psychosocial domain $(d f=2)$} & \multicolumn{3}{|c|}{ Physical domain $(d f=2)$} & \multicolumn{3}{|c|}{ Communication domain $(d f=2)$} \\
\hline & SBB & $F$ & $P$ & SBB & $F$ & $P$ & SBB & $F$ & $P$ \\
\hline Ignoring patient domain & 1122.44 & 2.89 & 0.056 & 2665.82 & 6.92 & 0.001 & 1746.78 & 4.51 & 0.011 \\
\hline Decision-making domain & 839.68 & 3.62 & 0.027 & 1766.18 & 7.69 & 0.001 & 1052.22 & 4.54 & 0.011 \\
\hline Professional competence domain & 311.76 & 3.68 & 0.026 & 572.02 & 6.80 & 0.001 & 570.33 & 6.79 & 0.001 \\
\hline
\end{tabular}

Table 3. Relationship among low, moderate, and high levels of moral distress domains and quality of care domains.

distress domains on each domain of care quality, multivariate analysis of variance and Fisher's exact test were used.

As Table 3 demonstrates, investigating the association among moral distress domains (ignoring patient, decision-making, and professional competence) and care quality domains (psychosocial, physical, and communication) demonstrated that ignoring patient did not have any relation with the psychosocial domain but it is related to the physical (bodily) and communication domains.

There was a significant correlation among the decision-making domain of moral distress and psychosocial, physical (bodily), and communication domains of care quality.

In addition, a statistically significant relationship was found between the professional competence domain of moral distress and psychosocial, physical, and communication domains of care quality.

Fisher's exact test (least significant difference) demonstrated that different levels of ignoring patient were not significantly associated with the psychological domain, but the nurses ignoring the patients more frequently, delivered moderate levels of physical care to the patients $(P=0.014)$, ignoring the patients more frequently led the nurses to the lowest levels of the communication domain $(P=0.014)$.

The nurses with low decision-making power had weak psychosocial domain $(P=0.006)$. The nurses that had high levels of decision-making power delivered moderate levels of physical care $(P=0.001)$, and the nurses with the highest decision-making power were highest in the communication domain $(P=0.04)$ and had a decreasing trend in the other groups.

The nurses with moderate levels of professional competence had higher levels of psychosocial $(P=0.032)$, physical $(P=0.003)$, and communication domains $(P=0.001)$.

\section{Discussion}

Considering that there were no articles that examine the relationship among each dimension of moral distress with each aspect of the care quality in detail of each dimension among these two variables separately, therefore, in this study, due to the limited resources, each dimension of moral distress with each aspect of the care quality has been discussed occasionally, in each dimension separately, and sometimes in general.

In the current study, ignoring patient had no effect on psychosocial domain but had effect on physical (bodily) and communication domains of patient care.

In Mohammadi's ${ }^{19}$ study, a strong, direct, and positive correlation was seen between patient-nurse relationship and patient care. This means that if the relationships between the nurses and patients are kept well, the patients assess and report nursing care positively and satisfyingly.

Therefore, establishing good relationships between patient-nurse by reducing the number of patients, increasing workforce and compliance with the standards by the system and managers will reduce ignoring the patient by nurses and then patient's charter would be followed. In addition, the decisions on some care are not confined to doctors and the nurses participate and then this phenomenon followed by training courses in special fields for this group to up-to-date their knowledge in this area.

Burhans's ${ }^{10}$ study demonstrated that the concept of nursing care quality, from the employed nurses' perspectives, is to satisfy humans' needs through care, sympathy, and respectful interactions in which responsibility, purposefulness, and support are the integral components of basic care.

Taking a look at Burhans's argument shows that the quality of clinical care increases with increasing nurses' attention to sympathy and support which in fact points to psychosocial, physical, and communication aspects.

According to Huffman, experiencing moral distress in hospital settings causes occurrence of numerous biological and psychological reactions and stress. Failure of organizations to create an environment in which moral distress is experienced, especially when nurses feel that they need to support patients' health in confronting organizational challenges, is a fact. ${ }^{20}$ In the present study, in accordance with Huffman's study, moral distress was an effective factor in the clinical settings which affected the psychosocial domain of quality care.

In our study, decision-making was effective on the psychosocial, physical (bodily), and communication 
domains of quality care. In Karanikola's ${ }^{21}$ study, the most frequent cause of moral distress was related to the poor cooperation between physicians and nurses and lack of paying attention to the nurses' ideas. According to Huffman's ${ }^{20}$ study, understanding the patient's suffering and pain is a result of medical decision-making which nurses have low power to influence and cooperate with. Unequal structures of power, which are common in institutes, intensify this problem.

CCU/ICU nurses need to detect moral distress and its adverse effect on providing optimal patient care. Nurses should be personally committed that their nursing care is not affected by moral distress and assume the role of leader in their wards to address this issue in the institutes they are working to decrease the effects of moral distress. ${ }^{20}$ Besides that, poor cooperation between the physicians and nurses and disregarding the nurses ideas have been reported in 17 European countries. ${ }^{22}$ The strategy of increasing the nurses power based on the available evidence and maximum cooperation of this population with the adopted decisions and implementation of standard treatments should be taken into account.

According to the findings of the current study, professional competence domain was directly correlated with psychosocial, physical (bodily), and communication domains of care quality. A review of 19 articles published between January 1984 and December 2011, given that relevant data were scant, demonstrated that the moral distress experienced by many nurses was related to difficult care conditions and feelings of depression, which could effect on professional situations and be in line with the current study; ${ }^{23}$ and these nurses are faced with certain problems in terms of the psychological domain of care quality. Rushton reported that when clinical nurses cannot convert moral choices into moral actions, they experience moral distress. The costs to reduce moral distress are high. Finally, moral distress, as an unresolved professional conflict, affects patient quality care,${ }^{24}$ suggesting interventions to remove distress and increase care, which is, in turn, an important factor for improving care quality.

In this study, the nurses experienced the most severe moral distress in providing care in unsafe conditions due to nursing understaffing, providing insufficient care to the patient, lack of professional competence, patient's bothering by the caregivers along with ignorance, and lack of reporting nursing errors. Milliken ${ }^{25}$ states that the people that do not have adequate executive ability to have an ethical performance develop moral distress. In Ameri's ${ }^{26}$ study, working with the nurses and other incompetent healthcare providers was an important factor for causing moral distress. In Fernandez's ${ }^{27}$ study, lack of observing patient safety principles by the nurse and other healthcare providers had the highest mean value. In Ebrahimi's ${ }^{28}$ study, consistent with Fernandez study, lack of observing patient safety principles by the nurse and other healthcare providers was the cause of the most severe distress and working with incompetent nurses and other incompetent healthcare providers had the greatest effect.

Most studies have reported lack of competence to be the cause of moral distress; however, in different studies, the addressees were different groups of healthcare providers, which is not specific to Iran. Available evidence indicates that this issue exists in other countries as well. Besides that, lack of separation of duties, nursing understaffing, and the nurses' heavy workload cause nurses' tiredom and lack of reporting nursing errors. Therefore, in the clinical setting bothering the patients especially by novice caregivers, due to mentioned reasons or fear of the consequences of medical errors becomes obvious and this incompetence exacerbates moral distress by affecting patient care.

In the current study, patient request for meeting with clergyman, introducing the new patient to the inpatients, familiarizing the patients with similar problems with each other, using fragrant substances to freshen the environment, and instructing and paying attention to religious ordinances in case of physical problems were reported to be low by the nurses.

Mohammadi's study demonstrated that among individual and social factors, nurses rush was the most important obstacle to prevent the establishment of communication followed by excessive patient expectations, nurse's unfamiliarity with the native language, nurse's unfamiliarity with the patients' rights, patient and nurse gender differences, problems outside the nurses' work environment, and age difference between the patient and the nurse are next barriers to communication. Besides that, the least important obstacle was class difference between the patient and the nurse. Regarding occupation characteristics, the nurses' physical and mental fatigue was the most important obstacle to establish the relationship, followed by heavy workload, hard work, lack of interest in your work, low pay and benefits, lack of information and skills, lack of amenities, and patient contacts with multiple nurses. The least important obstacle was nursing shift works. Regarding clinical conditions, the obstacles to establish the relationship with the patients was accompanying the patient, followed by the patient suffering from transmissible diseases, severe illness, and previous hospitalization. ${ }^{29}$

The effective factors in implementing nursing process and nursing care quality are various and complicated. None of the mentioned factors have precedence over the others and all of them are related to each other as a chain and should therefore be considered in parallel with each other so that clinical, psychosocial, and 
communication domains of care become more important to the nurses and the physicians and the managers pay attention to care quality more than ever, realize potential words and measures, and provide quality orientation background and consider the patients important. Besides that, because Islam, the Imams, and clergymen have a special status in Iran, the healthcare providers should pay further attention to the effect of communication therapy particularly for psychiatric patients and familiarizing the patients with similar problems with each other to strengthen the newly admitted patients' souls and speed up their recovery process.

Majidi's ${ }^{30}$ study showed that in the domain of different wards personnel activities, promotion of nurses' selfesteem in making independent decision was the most important. Conducting clinical care according to the clients' values and beliefs was the least important. ${ }^{30}$

Yaghobi's study showed that the openness of communication channels, feedback and communication about errors, the frequency of reporting incidents, employee affairs, and nonpunitive responses to errors had unpleasant status and needed to be seriously considered, particularly employee affairs and nonpunitive responses to errors that attained very low scores. But, certain dimensions such as teamwork in the unit, organizational learning, and continuous improvement were the strengths of patient care safety. ${ }^{31}$

It is recommended to use the nurses' ideas in decision-making and evaluate the performance according to the ethical principles and not only age, especially in the ICUs/CCUs, hold pre-internship and clinical competence exams before introduction of the clinical staff into bedside, increase the instruction of male nurses for increasing male patients comfort and implement compliance plan, pay further attention to the ratio of the nurses' number to the patients' number for eliminating fatigue, increase clinical qualifications and patient safety, implement the nursing process and provide principal and safe care for the patient rather than only report the evidence to attain the score assigned by insurance companies and other organizations, use native workforce especially nurses for maximizing their presence at the patient bedside and due to familiarity with the region language and culture, and make nursing fields, even postgraduate ones and prior to their introduction to the CCUs/ICUs, specialized before the standard evaluation and after attaining the points and attending courses and workshops as well as working in general wards as a clinical nurse serving as prerequisite for introduction to the CCUs/ICUs.

\section{Conclusions}

Moral distress affects the quality of care, adjustment and implementation of programs for reducing tension in all three dimensions (ignoring patients, decision-making, and professional competence) according to helpful and standard designs in the clinical setting have been emphasized and besides that patients' and healthcare providers' survey and application of constructive comments in the clinical setting is recommended. At the top of the organization's operational plan, to enhance quality in all three dimensions of care quality (psychosocial, physical, and communication), provide and maintain efficient and trained staff along with necessary and upto-date facilities, is proposed.

\section{Acknowledgments}

This study was part of research project, which has been approved by Tabriz University of Medical Sciences. Our appreciation goes to deputy officials in Tabriz University of Medical Sciences for their financial support, contribution, and all the nurses at the healthcare institutes and universities.

\section{Ethical approval}

Ethical issues are not involved in this paper.

\section{Conflicts of interest}

All contributing authors declare no conflicts of interest.

\section{References}

1. Ganske KM. Moral distress in academia. Online $J$ Issues Nurs. 2010;15:Manuscript 6.

2. The ICN code of ethics for nurses: revised 2012 (2012). International Council of Nurses. Web site. https//www.icn.ch/images/ stories/documents/about/icncode_english.pdf.

3. Epstein EG, Hamric AB. Moral distress, moral residue, and the crescendo effect. $J$ Clin Ethics. 2009;20:330-342.
4. Cavaliere TA, Daly B, Dowling D, Montgomery K. Moral distress in neonatal intensive care unit RNs. Adv Neonatal Care. 2010;10:145-156.

5. Mrayyan MT, Hamaideh SH. Clinical errors, nursing shortage and moral distress: the situation in Jordan. J Res Nurs. 2009;14:319-330.

6. Corley MC, Minick P, Elswick RK, Jacobs M. Nurse moral distress and ethical work environment. Nurs Ethics. 2005;12:381-390. 
7. Kelly B. Preserving moral integrity: a follow-up study with new graduate nurses. J Adv Nurs. 1998;28:1134-1145.

8. Mander R. Encouraging students to be research minded. Nurse Educ Today. 1988;8:30-35.

9. Redfern SJ, Norman IJ. Measuring the quality of nursing care: a consideration of different approaches. J Adv Nurs. 1990;15:1260-1271.

10. Burhans LM, Alligood M. Quality nursing care in the words of nurses. J Adv Nurs. 2010;66:1689-1697.

11. Driscoll A, Currey J, George M, Davidson PM. Changes in health service delivery for cardiac patients: implications for workforce planning and patient outcomes. Aust Crit Care. 2013;26:55-57.

12. Decker FH. Occupational and nonoccupational factors in job satisfaction and psychological distress among nurses. Res Nurs Health. 1997;20:453-464.

13. Fazljoo E, Borhani F, Abbaszadeh A, Razban F. The relationship between nurses' perceptions of moral distress and the ethical climate in Shahid Sadoughi University of Medical Sciences of Yazd. IJME. 2014;7:80-90.

14. Austin W, Lemermeyer G, Goldberg L, Bergum V, Johnson MS. Moral distress in healthcare practice: The situation of nurses. HEC Forum. 2005;17:33-48.

15. Ebrahimi H, Nikravesh M, Oskouie F, Ahmadi F. Stress: major reaction of nurses to the context of ethical decision making. Razi J Med Sci. 2007;14:7-15.

16. Ebrahimi $\mathrm{H}$, Namdar $\mathrm{H}$, Vahidi M. Relationship of Nurses Perception from Mental Illness Stigma and Quality of Nursing Care in Psychiatric Wards of Teaching Hospitals in North West of Iran. Iran: Nursing \& Midwifery School, Tabriz University of Medical Sciences; 2010.

17. Barlem ELD, Lunardi VL, Lunardi GL, TomaschewskiBarlem JG, Almeida AS, Hirsh CD. Psycometric characteristics of the Moral Distress Scale in Brazilian nursing professionals. Texto Contexto Enfermagem. 2014;23.

18. Joolaee S, Jalili HR, Rafii F, Hajibabaee F, Haghani H. Relationship between moral distress and job satisfaction among nurses of Tehran University of Medical Sciences Hospitals. J Hayat. 2012;18:42-51.

19. Mohammed MA, Odetola TD. Evaluation of patients' perception of nursing care in selected health institutions in Edo state, Nigeria. J Med Sci. 2014;5:12-19.
20. Huffman DM, Rittenmeyer L. How professional nurses working in hospital environments experience moral distress: a systematic review. Crit Care Nurs Clin North Am. 2012;24:91-100.

21. Karanikola MN, Albarran JW, Drigo E, et al. Moral distress, autonomy and nurse-physician collaboration among intensive care unit nurses in Italy. J Nurs Manage. 2013;14:1-13.

22. Papathanassoglou ED, Karanikola MN, Kalafati M, Giannakopoulou M, Lemonidou C, Albarran JW. Professional autonomy, collaboration with physicians, and moral distress among European intensive care nurses. Am J Crit Care. 2012;21:40-51.

23. Oh $Y$, Gastmans C. Moral distress experienced by nurses: A quantitative literature review. Nurs Ethics. 2015;22:15-31.

24. Rushton $\mathrm{CH}$. Defining and addressing moral distress: tools for critical care nursing leaders. AACN Adv Crit Care. 2006;17:161-168.

25. Milliken A. Nurse ethical sensitivity: an integrative review. Nurs Ethics. 2018;25: 278-303.

26. Ameri M, Safavibayat Z, Kavousi A. Evaluation of clinical situations leading to moral distress in nurses working in oncology wards of Tehran-based educational hospitals in 2012. Mod Care J. 2014;11:153-160.

27. Fernandez-Parsons R, Rodriguez L, Goyal D. Moral distress in emergency nurses. J Emerg Nurs. 2013;39:547-552.

28. Ebrahimi H, Kazemi A, Asghari Jafarabadi M, Azarm A. Moral distress in nurses working in educational hospitals of Northwest medical of Iran. Iran J Med Ethics Hist Med. 2014;6:80-88.

29. Mohammadi I, Mozafari M, Jamshid Beigi E, Kaikhavani S. Barriers to effective nurse- patient communication from perspective of nurses employed in educational hospitals of llam. J Neyshabur Univ Med Sci. 2014;2:20-27.

30. Majidi SA, Mehrabian F, Asgharnia M, Safar Mohammadi $\mathrm{H}$, Kazemnezhad E. Effective factors on adaptation of theoretical education and practical nursing care. Res Med Educ. 2015;7:12-20.

31. Yaghobi Far MA, Takbiri A, Haghgoshaye E, Tabarraye $Y$. The survey of patient safety culture and recognizing its weaknesses and strengths in Sabzevar Hospitals: 2011. J Sabzevar Univ Med Sci. 2013;20:154-164. 